\title{
Segurança alimentar em domićlios chefiados por idosos, Brasil
}

\author{
Foodsearityinhaseh dosheadedby dderly, Brazil
}

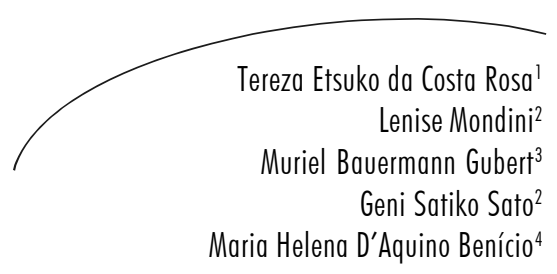

Resumo

A segurança alimentar nos domicílios chefiados por idosos tem uma dimensão especial no que se refere às condições de saúde e bem-estar, uma vez que parece evidente a importância de garantir a este contingente populacional a possibilidade de continuar a contribuir na sociedade de forma ativa e produtiva. Objetivo: Determinar a prevalência de insegurança alimentar em domicílios cujos chefes são idosos, segundo características sociodemográficas. Métodos: Trata-se de estudo descritivo com domicílios cujos chefes têm 60 anos ou mais de idade declarada, selecionados da Pesquisa Nacional de Amostra de Domicílios - PNAD 2004. Empregou-se a Escala Brasileira de Insegurança Alimentar, classificando-se os domicílios em segurança alimentar e insegurança alimentar leve, moderada e grave. A análise descritiva dos dados incluiu a distribuição de frequência dos domicílios de acordo com os níveis de insegurança alimentar nos estratos das variáveis sociodemográficas, levando-se em consideração o efeito do desenho. Resultados: O estudo mostrou que $29,8 \%$ dos domicílios se encontravam na condição de insegurança alimentar e que tal condição estava significativamente associada com regiões menos abastadas (Norte/Nordeste, rural), com os segmentos populacionais mais desfavorecidos (mais pobres e menos escolarizados) e, ainda com características de gênero (mulheres) e raciais (indígenas, pardos e pretos) as quais sabidamente ocupam os níveis inferiores da hierarquia social. Conclusão: A distribuição da insegurança alimentar em domicílios chefiados por idosos segue tendência similar dos domicílios

\footnotetext{
1 Instituto de Saúde. Secretaria de Estado da Saúde de São Paulo. São Paulo, SP, Brasil.

2 Instituto de Economia Agrícola. Secretaria da Agricultura e Abastecimento de São Paulo. São Paulo, SP, Brasil.

3 Faculdade de Ciências da Saúde, Departamento de Nutrição. Universidade de Brasília. Brasília, DF - Brasil

4 Faculdade de Saúde Pública, Departamento de Nutrição. Universidade de São Paulo. São Paulo, SP, Brasil.
}

\author{
Correspondência / Correspondence \\ Tereza Etsuko da Costa Rosa \\ Instituto de Saúde \\ Secretaria de Estado da Saúde de São Paulo \\ Rua Santo Antônio, 590 \\ 01314-000 São Paulo, SP, Brasil \\ E-mail: tererosa@isaude.sp.gov.br
}

Palavras-chave: Idosos. Fatores socioeconômicos. Qualidade de vida. Comportamento alimentar. Insegurança alimentar. 
brasileiros, ratificando a maior prevalência desta condição nos estratos socioeconômicos mais desfavorecidos da população ou entre características associadas à pobreza.

\section{Abstract}

Food security in households headed by elderly people has special dimension as for health and welfare, since it is clearly important to ensure that they go on contributing to society in an active and productive way. Objective: To determine the prevalence of food insecurity in households whose heads are older, according to socio-demographic characteristics. Methods: It is a descriptive study with households whose heads have 60 years of age or over declared, selected from the National Sample Household Survey - PNAD 2004. We applied the Brazilian Food Insecurity Scale, ranking the households into food security and food insecurity, moderate and severe. Descriptive analysis of data included the frequency distribution of households according to levels of food insecurity in the strata of socio-demographic variables, taking into account the design effect. Results: The study showed that $29.8 \%$ of households were in the condition of food insecurity and that this condition was significantly associated with less affluent regions (north / northeast, rural), with the most vulnerable population segments (the poorest and least educated) and also with characteristics of gender (women) and race (Indian, mulatto and black) which is known to occupy the lower levels of social hierarchy. Conclusion: The distribution of food insecurity in households headed by elderly people, following a similar trend of Brazilian households, confirms the higher prevalence of this condition in socioeconomic disadvantaged population or characteristics associated with poverty.
Key words: Elderly. Socioeconomic factors. Quality of life. Feeding behavior. Food insecurity.

\section{INTRODUÇÃO}

Segurança Alimentar e Nutricional é a garantia do direito de todos ao acesso a alimentos de qualidade, em quantidade suficiente e de modo permanente, com base em práticas alimentares saudáveis e respeitando as características culturais de cada povo, manifestadas no ato de se alimentar. Esta condição não pode comprometer o acesso a outras necessidades essenciais, nem sequer $\mathrm{o}$ sistema alimentar futuro, devendo se realizar em bases sustentáveis. É responsabilidade dos Estados nacionais assegurarem este direito e devem fazêlo em obrigatória articulação com a sociedade civil, dentro das formas possíveis para exercê-lo. ${ }^{1}$

Três elementos básicos compõem o conceito de segurança alimentar: "quantidade suficiente, regularidade e qualidade”, assumindo uma abordagem ímpar quando se trata de garantir alimentação adequada aos idosos, grupo populacional cujo crescimento é fenômeno mundial. ${ }^{2} \mathrm{~A}$ insegurança alimentar entre idosos está associada com estado nutricional e de saúde inadequados, ${ }^{3}$ que podem contribuir para o aumento da ocorrência de doenças, dependência para atividades da vida diária, redução da resistência a infecções e aumento de hospitalização. ${ }^{4,5}$

No Brasil, somente em uma década, a população de idosos (60 anos ou mais de idade), segundo o Estatuto do Idoso 6 aumentou 35,5\% entre 1991 e 2000 e, em 2008 já representava 11,1\% da população total. ${ }^{7,8}$ É importante destacar que, segundo dados do Censo 2000 (último disponível), naquele ano $62,4 \%$ dos idosos eram responsáveis pelos domicílios brasileiros. ${ }^{7}$ Ora, neste sentido, a segurança alimentar nesses domicílios tem uma dimensão especial no que se refere às condições de saúde e bem-estar do idoso, uma vez que parece evidente a importância de 
garantir a esse contingente populacional a possibilidade de continuar contribuindo na sociedade de forma ativa e produtiva.

O presente estudo tem por objetivo determinar a prevalência de insegurança alimentar em domicílios cujos chefes são idosos, segundo características sociodemográficas. Espera-se, desta forma, contribuir para o conhecimento da situação de segurança alimentar da população idosa brasileira, em especial aquela responsável pelos domicílios, como uma questão de interesse para a adequação de políticas públicas direcionadas a este segmento populacional.

\section{METODOLOGIA}

Trata-se de estudo descritivo com domicílios cujos chefes tinham 60 anos ou mais de idade declarada, selecionados da Pesquisa Nacional de Amostra de Domicílios (PNAD 2004), executada pelo Instituto Brasileiro de Geografia e Estatística (IBGE), compondo uma amostra de 23.877 domicílios em todo o Brasil. ${ }^{9}$

A mensuração de segurança/insegurança alimentar foi realizada por meio de inquérito domiciliar com aplicação de questionário junto a um adulto que soubesse responder sobre a alimentação da família. O instrumento de coleta de dados empregado foi a Escala Brasileira de Insegurança Alimentar (EBIA), com 15 perguntas referentes à experiência de insuficiência alimentar percebida nos últimos 90 dias que antecedem a entrevista. A condição de segurança alimentar pode ser classificada em quatro categorias: segurança alimentar e insegurança alimentar leve, moderada e grave, correspondendo à pontuação atribuída a cada domicílio, referente ao número de respostas afirmativas às perguntas da escala, diferenciada para domicílios com pelo menos um morador com idade inferior a 18 anos e domicílios com todos os moradores com 18 anos ou mais de idade. ${ }^{9,10}$
Os domicílios foram identificados segundo os níveis de insegurança alimentar nas grandes regiões do país, situação do domicílio (urbano e rural), rendimento domiciliar per capita mensal, número de pessoas no domicílio, além do nível de escolaridade, sexo e raça/cor do chefe da família.

A análise descritiva dos dados incluiu a distribuição de frequência dos domicílios de acordo com os níveis de insegurança alimentar nos estratos das variáveis sociodemográficas, com correção para o efeito do desenho," utilizando-se o módulo específico Survey (família de comandos syy) do software Stata 8.2, que permite calcular variâncias em amostras complexas de acordo com o processo amostral adotado na PNAD. As associações da variável de interesse (segurança/ insegurança alimentar total) com as demais variáveis foram verificadas por meio da estatística "F", levando-se em consideração o efeito do desenho, adotando-se nível de significância de 5\%. Os dados foram processados e analisados nos softwares SPSS versão 13.0 e Stata versão 8.0, respectivamente.

Por se tratar de estudo originado de análise de banco de dados de uso e acesso público (PNAD 2004), este não foi submetido à apreciação em Comitê de Ética em Pesquisa e não existiu nenhum tipo de conflito de interesses para a elaboração do artigo.

\section{RESULTADOS}

A tabela 1 mostra a distribuição de frequência de segurança alimentar e de insegurança alimentar (total), além dos níveis leve, moderado e grave em domicílios cujos chefes eram pessoas com 60 anos e mais de idade, de acordo com variáveis sociodemográficas. Também se verificou a associação da variável "insegurança alimentar total” (sim/não) com cada uma das demais variáveis, expressa pelos níveis de significância do teste (valor $\mathrm{p}$ ).

O módulo survey do Stata permite o cálculo de variâncias em amostras complexas, uma vez que o sorteio em diferentes estágios retira a equiprobabilidade de sorteio que ocorre ao se considerar o processo de amostragem casual simples. 
Regionalmente, a situação de insegurança alimentar foi mais prevalente nas regiões Norte e Nordeste do país, onde níveis de insegurança alimentar moderado ou grave foram detectados em mais de 1/4 dos domićlilios. Situação oposta ocorreu nas regiões Sul e Sudeste, onde tais condições só foram encontradas em cerca de 10\% dos domicílios e a grande maioria (80\%) deles foi classificada como em situação de segurança alimentar. Os resultados indicam que existe associação entre todos os tipos de insegurança alimentare região onde se localizavam os domicílios $(\mathrm{p}<0,001)$.
Embora as prevalências de insegurança alimentar nas zonas urbana e rural não sejam marcadamente diferentes, conforme verificado nas grandes regiões, a prevalência de domicílios em situação de insegurança alimentar na zona rural é maior em relação à urbana $(32,2 \%$ contra 29,3\%, respectivamente; $\mathrm{p}=0,037$ ).

No que diz respeito ao gênero, os domicílios chefiados pelos homens se encontravam em melhor situação de segurança alimentar em relação àqueles chefiados por mulheres $(\mathrm{p}<0,001)$.

Tabela 1 - Prevalência (\%) de insegurança alimentar em domicílios cujos chefes são idosos, segundo variáveis sociodemográficas. Brasil, PNAD - 2004.

\begin{tabular}{|c|c|c|c|c|c|c|}
\hline \multirow[b]{2}{*}{ Variáveis } & \multirow[b]{2}{*}{$\mathrm{n}$} & \multirow{2}{*}{$\begin{array}{l}\text { Segurança } \\
\text { alimentar }\end{array}$} & \multicolumn{4}{|c|}{ Insegurança Alimentar } \\
\hline & & & Total & Leve & Moderada & Grave \\
\hline \multicolumn{7}{|l|}{ Regiões } \\
\hline Norte & 2208 & 55,8 & 44,2 & 16,2 & 16,7 & 11,3 \\
\hline Nordeste & 7571 & 54,0 & 46,0 & 16,8 & 19,4 & 9,8 \\
\hline Centro-Oeste & 2092 & 71,5 & 28,5 & 12,2 & 11,2 & 5,1 \\
\hline Sudeste & 8028 & 78,3 & 21,7 & 11,0 & 7,6 & 3,1 \\
\hline $\begin{array}{l}\text { Sul } \\
p<0,001\end{array}$ & 3978 & 78,9 & 21,1 & 9,8 & 7,3 & 4,0 \\
\hline \multicolumn{7}{|l|}{ Área } \\
\hline Urbana & 19836 & 70,7 & 29,3 & 12,6 & 11,2 & 5,5 \\
\hline $\begin{array}{l}\text { Rural } \\
p=0,037\end{array}$ & 4041 & 67,8 & 32,2 & 13,4 & 12,7 & 6,1 \\
\hline \multicolumn{7}{|l|}{ Gênero } \\
\hline Masculino & 14410 & 72,2 & 27,8 & 12,3 & 10,5 & 5,0 \\
\hline $\begin{array}{l}\text { Feminino } \\
p<0,001\end{array}$ & 9467 & 67,0 & 33,0 & 13,5 & 13,0 & 6,5 \\
\hline \multicolumn{7}{|l|}{ Raça (Cor) } \\
\hline Indígena & 51 & 45,1 & 54,9 & 20,9 & 27,0 & 7,0 \\
\hline Branca & 12837 & 79,1 & 20,9 & 10,2 & 7,5 & 3,2 \\
\hline Negra & 1863 & 56,4 & 43,6 & 17,0 & 18,3 & 8,3 \\
\hline Amarela & 120 & 90,5 & 9,5 & 6,2 & 1,4 & 1,9 \\
\hline $\begin{array}{l}\text { Parda } \\
p<0,001\end{array}$ & 9005 & 57,8 & 42,2 & 16,2 & 16,9 & 9,1 \\
\hline \multicolumn{7}{|l|}{$\begin{array}{l}\text { Renda per } \\
\text { capita }\end{array}$} \\
\hline$<1 / 4$ & 592 & 21,4 & 78,6 & 17,5 & 27,0 & 34,1 \\
\hline $1 / 4-1 / 2$ & 2097 & 30,3 & 69,7 & 22,7 & 30,3 & 16,7 \\
\hline $1 / 2-1$ & 5536 & 52,8 & 47,2 & 20,6 & 19,4 & 7,2 \\
\hline $\begin{array}{l}>=1 \\
p<0,001\end{array}$ & 15652 & 82,5 & 17,5 & 8,7 & 6,0 & 2,8 \\
\hline
\end{tabular}


Tabela 1 - Prevalência (\%) de insegurança alimentar em domicílios cujos chefes são idosos, segundo variáveis sociodemográficas. Brasil, PNAD - 2004. (Continuação)

\begin{tabular}{|c|c|c|c|c|c|c|}
\hline \multirow[b]{2}{*}{ Variáveis } & \multirow[b]{2}{*}{$\mathrm{n}$} & \multirow{2}{*}{$\begin{array}{l}\text { Segurança } \\
\text { alimentar }\end{array}$} & \multicolumn{4}{|c|}{ Insegurança Alimentar } \\
\hline & & & Total & Leve & Moderada & Grave \\
\hline \multicolumn{7}{|c|}{$\begin{array}{l}\text { Escolaridade } \\
\text { (anos) }\end{array}$} \\
\hline$<1$ & 8639 & 55,9 & 44,1 & 16,7 & 17,8 & 9,6 \\
\hline $1-3$ & 5052 & 69,8 & 30,2 & 12,9 & 12,1 & 5,2 \\
\hline $4-7$ & 5650 & 77,2 & 22,8 & 11,4 & 7,9 & 3,5 \\
\hline $7-10$ & 1343 & 83,4 & 16,6 & 9,6 & 4,9 & 2,1 \\
\hline $\begin{array}{l}>10 \\
p<0,001\end{array}$ & 3181 & 92,2 & 7,8 & 4,9 & 2,2 & 0,7 \\
\hline \multicolumn{7}{|c|}{$\begin{array}{l}\mathrm{N}^{\circ} \text { de pessoas } \\
\text { no domicílio }\end{array}$} \\
\hline Sozinho & 4582 & 76,4 & 23,6 & 8,9 & 8,6 & 6,1 \\
\hline 2 & 7094 & 76,8 & 23,2 & 9,6 & 9,2 & 4,4 \\
\hline $3-4$ & 7815 & 69,1 & 30,9 & 14,1 & 11,9 & 4,9 \\
\hline $\begin{array}{l}5 \mathrm{e}+ \\
p<0,001\end{array}$ & 4386 & 53,1 & 46,9 & 20,1 & 18,2 & 8,6 \\
\hline Total & 23877 & 70,2 & 29,8 & 12,7 & 11,5 & 5,6 \\
\hline
\end{tabular}

A prevalência de insegurança alimentar segundo a cor da pele ou raça do chefe do domicílio mostra que a pior situação foi encontrada entre os indígenas ( $34 \%$ de tipo moderado ou grave) e entre pretos e pardos ( $26 \%$ de tipo moderado ou grave).

No que se refere à renda domiciliar per capita, escolaridade e número de pessoas residentes no domicílio, além de sua associação com a condição de insegurança alimentar, observou-se um gradiente da distribuição da variável de interesse segundo os estratos de cada uma das variáveis independentes referidas.

Relação claramente inversa foi verificada entre o nível socioeconômico, aqui representado pela renda domiciliar per capita mensal e escolaridade do chefe do domicílio e a condição de insegurança alimentar (teste de tendência linear $\mathrm{p}<0,0001$ ). Entre os domicílios com renda per capita maior que um salário mínimo, nota-se $2,8 \%$ de insegurança alimentar grave, enquanto que tais proporções se elevaram rapidamente para $7,2 \%$, $16,7 \%$ e $34,1 \%$ à medida que o nível de renda se reduz. Tendência semelhante foi observada nos diversos níveis de escolaridade, não obstante as proporções de insegurança alimentar grave sejam de menor magnitude.

A condição de insegurança alimentar apresentou relação direta com o número de pessoas no domicílio (teste de tendência linear $\mathrm{p}<$ 0,0001). Maiores proporções de insegurança alimentar em níveis moderado ou grave foram observadas quando o domicílio era composto por três a quatro pessoas $(16,8 \%)$ e por cinco ou mais pessoas $(26,8 \%)$. Desta forma, cerca de $3 / 4$ dos domicílios com no máximo dois moradores apresentaram situação de segurança alimentar.

\section{DISCUSSÃO}

A partir da classificação dos domicílios, exclusivamente chefiados por pessoas com 60 anos ou mais de idade, de acordo com a segurança alimentar, investigada por meio do levantamento suplementar da Pesquisa Nacional por Amostra 
de Domicílios (PNAD 2004) sobre Segurança Alimentar (EBIA), o estudo mostrou que 70,2\% desses domicílios se encontravam em condição de segurança alimentar. $\mathrm{O}$ estudo evidenciou também que a condição oposta $(29,8 \%)$ estava associada às regiões menos abastadas (Norte/ Nordeste, rural), com os segmentos populacionais mais desfavorecidos (mais pobres e menos escolarizados) e, ainda, com características de gênero (mulheres) e raciais (indígenas, pardos e pretos), as quais sabidamente ocupam os níveis inferiores da hierarquia social.

A validade da escala, confirmada em estudos anteriores ${ }^{10-12} \mathrm{e}$ aliada ao planejamento amostral empreendido na PNAD, ${ }^{9}$ permitiu estimativas confiáveis de prevalência de insegurança alimentar no país, bem como sua associação com alguns aspectos sociodemográficos e econômicos, com focalização nos domicílios que tinham uma pessoa idosa como referência.

A aferição da insegurança alimentar, quando feita pela EBIA, engloba forte influência do componente de acesso financeiro ao alimento, uma vez que a maior parte das perguntas da escala se refere à condição "ter dinheiro suficiente" para a aquisição do alimento. ${ }^{13}$

A distribuição da insegurança alimentar em domicílios chefiados por idosos seguiu a mesma tendência dos domicílios brasileiros, sendo a insegurança alimentar moderada/grave maior também nas regiões Norte $(31,1 \%)$ e Nordeste $(37,8 \%) .{ }^{9}$ De fato, no início da presente década, o rendimento médio mensal das pessoas com 60 anos e mais de idade, responsáveis pelos domicílios, era quase o dobro nas regiões do centro-sul do país, quando comparado ao das regiões Norte e Nordeste e, de 2,5 vezes entre residentes de área urbana comparado aos de área rural. ${ }^{7}$

Em domicílios situados na zona rural, elevadas prevalências de insegurança alimentar já foram observadas em outros estudos. Parte deste fenômeno pode ser explicada pelo fato de que a referência de ausência de renda para aquisição de alimentos nas áreas rurais foi mais frequente quando a condição de insegurança alimentar se fazia presente. ${ }^{14}$

Hoffmann \& Kageyama ${ }^{15}$ relacionam a insegurança alimentar com a pobreza no Brasil, ressaltando que a pobreza afeta $65,1 \% \mathrm{da}$ população rural e $29,2 \%$ da população urbana, sendo que $9,2 \%$ da população na área rural vivem em estado de extrema pobreza. Estudos mostram, entretanto, que excluindo-se o problema da renda, a zona rural apresenta características mais favoráveis à segurança alimentar, como maior frequência de produção alimentar para autoconsumo e disponibilidade de alimentos pela prática extrativista. ${ }^{16}$

A insegurança alimentar é mais prevalente entre as mulheres responsáveis por domicílios, comparadas aos homens na mesma condição. Dados do último Censo indicam que cerca de 40\% dos domicílios chefiados por idosos eram de mulheres, das quais um terço morava sozinha, contra $9,5 \%$ dos homens, fato decorrente do diferencial de expectativa de vida entre os sexos. ${ }^{7}$ As mulheres constituem a maioria dos pobres, entre idosos e não-idosos; particularmente entre as idosas, têm menos experiência de trabalho no mercado formal e apresentam baixa escolaridade, o que pode implicar menor renda, ${ }^{7,17}$ conforme verificado no ano de realização da PNAD, quando o rendimento médio das mulheres era cerca de um terço menor que o rendimento auferido pelos homens. ${ }^{18}$

Em relação à raça/cor, a pior situação na ocorrência de insegurança alimentar entre os indígenas ( $34 \%$ de tipo moderado ou grave) e entre pretos e pardos ( $26 \%$ de tipo moderado ou grave) foi semelhante aos dados encontrados em outros estudos.

No Brasil, os povos indígenas estão expostos a transformações ambientais e socioeconômicas, que os colocam em situação de alta vulnerabilidade à insegurança alimentar. ${ }^{19}$ Entre a população cujo responsável é pardo ou negro, normalmente todos os indicadores sociodemográficos são piores, ${ }^{8}$ ressaltando-se que o percentual de pobres nestes grupos com 60 anos 
ou mais de idade era o dobro da população branca nesta faixa etária ao final da década de 1990.17

Conforme esperado, ratificando o panorama já analisado, a condição de insegurança alimentar das famílias com chefes idosos apresentou-se mais prevalente nos estratos socioeconômicos mais desfavorecidos da população brasileira.

Um dos determinantes proximais da condição de insegurança alimentar é a desigualdade de renda, juntamente com a proxy (aproximação) escolaridade. $\mathrm{Na}$ análise da insegurança em domicílios chefiados por idosos, a renda e escolaridade do chefe mostraram-se inversamente proporcionais às prevalências de insegurança alimentar. Nota-se que a proporção de domicílios com os menores rendimentos alcançou 12 vezes a proporção de insegurança alimentar, comparados aos de maiores rendimentos. Por outro lado, mesmo nas famílias cujas rendas, em tese, são consideradas plenamente suficientes para a satisfação das necessidades nutricionais das pessoas (renda per capita $>1$ salário mínimo), persistem prevalências da ordem de $6,0 \%$ e de $8,7 \%$ de insegurança alimentar moderada e leve, respectivamente. Isso reforça a tese de alguns estudiosos ${ }^{15}$ de que, embora seja inegável a íntima relação entre satisfação das necessidades básicas e disponibilidade de renda, outras dimensões devem ser consideradas também para a sobrevivência das pessoas e famílias.

A baixa escolaridade do responsável se constitui num parâmetro importante para a condição de insegurança alimentar no domicílio. Em geral, o número de anos de estudo agrega valores que podem facilitar o acesso à informação, a serviços públicos etc., além do fato de que pouca escolaridade, via de regra, se associa à baixa renda.

Conforme verificado no estudo sobre a insegurança alimentar nos domicílios brasileiros como um todo, mesmo depois de controlado o efeito da renda, a escolaridade da pessoa de referência contribui, significativamente, para reduzir a probabilidade de insegurança alimentar. ${ }^{20}$

No entanto, vale considerar que a condição de insegurança alimentar é ainda pior nas famílias cujos chefes são indivíduos com menos de 60 anos de idade $(36,2 \%$ contra 29,8\%; $\mathrm{p}<0,001$ - dados não apresentados), mesmo nos níveis mais graves. Isso mostra que o idoso, arrimo de família, pode gerar uma situação de proteção em relação à insegurança alimentar. Segundo dados de 2008, ${ }^{8}$ em metade dos domicílios com idosos, a renda destes representava a maior parte $\mathrm{da}$ renda familiar, oriunda principalmente de aposentadoria, que garante renda estável e maior segurança econômica.

No município de Campinas-SP, um estudo sobre insegurança alimentar em famílias selecionadas com moradores com 65 anos ou mais de idade revelou que cerca de $70 \%$ delas era chefiada pelo indivíduo idoso com contribuição substancial para a renda familiar. ${ }^{12}$ Neste sentido, especificamente para a população responsável pelo domicílio, embora a renda média dos idosos seja inferior à da população maior de dez anos de idade, seu crescimento, no período entre 1991 e 2000 , foi muito mais expressivo (63\% contra $42 \%$, respectivamente). Ressalta-se ainda, neste período, que o crescimento no rendimento médio do idoso residente em área rural foi maior do que o dos responsáveis não idosos $(77 \%$ contra $53 \%)^{7}$

A política brasileira de seguridade social, que garantiu uma cobertura praticamente universal $(87,2 \%),{ }^{21}$ parece ter grande influência nesse panorama. De fato, em 2002, $73 \%$ do total das transferências de renda do Governo Federal foram gastos com aposentadorias e pensões, enquanto apenas $1,5 \%$ dos recursos beneficiaram as famílias pobres por meio dos Programas de Renda Mínima. ${ }^{22}$

Tais estatísticas, supostamente favorecedoras dos idosos, geraram um debate em torno do que vem sendo denominado "viés intergeracional". ${ }^{23}$ Não obstante outros estudiosos das políticas sociais no Brasil sustentem que faltam evidências definitivas sobre a existência de conflito entre as gerações, ${ }^{24}$ certamente a universalização dos benefícios de seguridade social resultou em melhorias nos padrões de vida da população mais envelhecida. 


\section{CONCLUSÕES}

Caberiam outros estudos sobre a condição de segurança alimentar da população idosa no contexto de domicílios em que outra pessoa, que não o idoso, seja o chefe da família, e nos quais talvez fossem reveladas configurações diversas das constatadas no presente estudo. Todavia, os determinantes da insegurança alimentar não devem diferir em sua natureza entre os domicílios chefiados por pessoas com 60 anos ou mais e aqueles que não o são. Portanto, a definição de prioridades e ajustes de medidas para a redução da insegurança alimentar, de acordo com as necessidades do contexto específico, deverá subordinar-se à

\section{REFERÊNCIAS}

1. Brasil. Lei $\mathrm{n}^{\circ}$ 11.346, de 15 de setembro de 2006. Cria o Sistema Nacional de Segurança Alimentar e Nutricional com vistas em assegurar o direito humano à alimentação adequada e dá outras providências. Diário Oficial da União 17 set 2006.

2. Wolfe WS, Frongillo EA, Valois P. Understanding the experience of food insecurity by elders suggests ways to improve its measurement. J Nutr 2003; 133 : 2762-69.

3. Lee JS, Frongillo Jr EA. Nutritional and health consequences are associated with food insecurity among US elderly persons. J Nutr 2001; 131:1503-9.

4. Reuben DB, Freendale GA, Harrison GF. Nutrition screening in older persons. J Am Geriatr Soc 1995; 43 : 415-25.

5. Jensen GL, Friedmann JM, Colemann CD, Smiciklas-Wrigth H. Screening for hospitalization and nutritional risks among community-dwelling older persons. Am J Clin Nutr 2001; 74:201-5.

6. Estatuto do Idoso. Lei $\mathrm{n}^{\mathrm{o}} 10.741$, de $1^{\circ} \mathrm{de}$ outubro de 2003. [Acesso em $30 \mathrm{abr}$ 2011].Disponível em: http:// www.planalto.gov.br/ccivil_03/Leis/2003/ L10.741.htm.

7. Brasil. Ministério do Planejamento, Orçamento e Gestão. Instituto Brasileiro de Geografia e Estatística (IBGE). Perfil dos idosos magnitude do efeito dos fatores sociodemográficos e regionais.

$\mathrm{Na}$ medida em que os resultados aqui discutidos apontam asfamílias pobres, das áreas rurais, como parte significativa dos domicílios afetados pela insegurança alimentar, políticas públicas impactantes nesse âmbito poderiam ser aquelas direcionadas à criação de programas que estimulem os idosos a desempenharem papel ativo na produção de alimentos. Programas que, aliados a políticas sociais e de saúde que incentivem a disponibilização de uma gama de serviços preventivos, de recuperação edesenvolvimento para as pessoasidosas, devem lhes permitirlevaruma vida omais independente possível em seu próprio lar e em sua comunidade, e continuar a ser cidadãos ativos e úteis à sociedade.

responsáveis pelos domicílios no Brasil 2002: Estudos e Pesquisas. Rio de Janeiro: IBGE; 2002. 97p.

8. Brasil. Instituto Brasileiro de Geografia e Estatística (IBGE). Pesquisa Nacional por Amostra de Domicílios : síntese de indicadores. 2008. [ Acesso em 10 Mar 2010]. Disponível em: www.ibge.gov.br .

9. Brasil. Ministério do Planejamento, Orçamento e Gestão. Instituto Brasileiro de Geografia e Estatística(IBGE). Segurança alimentar : pesquisa nacional por amostra de domicílios. Rio de Janeiro: IBGE; 2006. 140p.

10. Segall-Corrêa AM, et al. Relatório Técnico: acompanhamento e avaliação da segurança alimentar de famílias brasileiras: validação de metodologia e de instrumento de coleta de informação. Campinas: Unicamp; 2004.

11. Perez-Escamilla $R$, et al. An adapted version of the U.S. Department of Agriculture Food Insecurity Module is a valid tool for assessing household food insecurity in Campinas, Brazil. J Nutr, Bethesda 2004 aug;134(8): 1923-1928.[ Acesso em 03 Mar 2010]. Disponível em: http:// jn.nutrition.org/cgi/reprint/134/8/1923.

12. Marín-León L, et al. A percepção de insegurança alimentar em famílias com idosos em Campinas. Cad Saúde Pública 2005; 21 (5): 1433-40.

13. Segall-Corrêa AM, et al. Evaluation of household insecurity in Brazil: validity 
assessment in diverse sociocultural settings. Santiago: Premio Redsan - FAO; 2008.

14. Vianna RPT, Segall-Corrêa AM. Insegurança alimentar das famílias residentes em municípios do interior do estado da Paraíba. Rev Nutr. 2008; 21: 111-122S.

15. Hoffmann R, Kageyama A. Pobreza, insegurança alimentar e pluriatividade no Brasil. Fortaleza: SOBER; 2007.

16. Yuyama LKO, et al. Segurança/Insegurança alimentar em famílias urbanas e rurais no estado do Amazonas: I. Validação de metodologia e de instrumento de coleta de informação. Acta amazônica. 2007; 37(2): 247252.

17. Barros RP, Mendonça R, Santos D. Incidência e natureza da pobreza entre idosos no Brasil: texto para discussão. Brasília: IPEA; 1998. 686p.

18. Brasil. Ministério do Planejamento, Orçamento e Gestão. Instituto Brasileiro de Geografia e Estatística (IBGE). Pesquisa Nacional por
Amostra de Domicílios.Rio de Janeiro: IBGE; 2005.

19. Fávaro T, Ribas DLB, Zorzatto JR, Segall-Corrêa AM, Panigassi G. Segurança alimentar em famílias indígenas.Cad. Saúde Pública 2007; 23(4): 785-793.

20. Hoffmann R. Determinantes da insegurança alimentar no Brasil: análise dos dados da PNAD de 2004. Rev SAN. 2008; 15(1): 49-61.

21. Camarano AA, Pasinato MT. Envelhecimento, pobreza e proteção social na América Latina. Rio de Janeiro: Ipea; 2007

22. Brasil. O gasto social do governo central: $2001 \mathrm{e}$ 2002. Brasília: Ministério da Fazenda; 2003.

23. Barros RP, Carvalho M. Desafios para a política social brasileira. Rio de Janeiro: Ipea; 2007

24. Goldani AM. Relações intergeracionais e reconstrução do estado de bem-estar. Por que se deve repensar essa relação para o Brasil? In: Camarano AA. Os novos idosos brasileiros: muito além dos 60? Rio de Janeiro: Ipea; 2004. p. 353-410. 
